\title{
MICROORGANISMOS DEL SUELO EN BOSQUES DE ÑIRE EN PATAGONIA SUR RESULTADOS PRELIMINARES
}

\author{
Gargaglione, Verónica ${ }^{4,5}$; Gonzalez Polo, Marina ${ }^{6}$ y Peri, Pablo Luis , $^{4,6}$
}

\section{RESUMEN}

Nothofagus antarctica (ñire) es una especie nativa del bosque andino-patagónico que se extiende desde los $36^{\circ} 30^{\prime}$ hasta los 56 $00^{\prime}$ LS. Es la segunda especie en importancia de la provincia de Santa Cruz, Argentina.

En estos bosques, como en la mayoría de los ecosistemas naturales, la descomposición de la materia orgánica es un importante proceso mediado por organismos heterótrofos que utilizan al material orgánico muerto, o detritus, como hábitat y fuente de carbono (C) y energía.

Actualmente, el $90 \%$ de estos bosques en Patagonia Sur están siendo utilizados como sistemas silvopastoriles, donde en una misma unidad de superficie coexisten el bosque, el estrato herbáceo y el ganado ovino o vacuno. Por ello, el objetivo del presente estudio fue generar información de base en cuanto a la actividad de los microorganismos de los bosques primarios de $N$. antarctica y bajo uso silvopastoril, a fin de poder detectar si este tipo de uso afecta a la actividad microbiana y por ende a la biología del suelo.

En primavera y verano se tomaron muestras de suelo $(5-10 \mathrm{~cm}$ de profundidad) en tres estancias distintas del Sur de la provincia de Santa Cruz (Cancha Carreras, Tres Marías y Morro Chico) que tienen bosque de ñire sin uso y bajo uso silvopastoril.

Se utilizó el método de fumigación-extracción para la detección del $\mathrm{C}$ en biomasa microbiana. No se encontraron diferencias significativas en el $\mathrm{C}$ contenido en la biomasa microbiana según el uso del bosque, ni la época del año (primavera o verano). El bosque primario contenía $1.209 \mu \mathrm{g}$ de $\mathrm{C}$ en biomasa microbiana por gramo de suelo seco, mientras que el bosque bajo uso silvopastoril presentó $850 \mu \mathrm{g}$ de $\mathrm{C}$.

El presente estudio es el primero que evalúa los microorganismos en los bosques silvopastoriles de ñire al Sur de Santa Cruz por lo cual la información generada es muy relevante.

El hecho de que el suelo sea un ambiente tan variable pudo haber influenciado para que no se encuentren diferencias significativas según el uso del bosque. En este sentido, es importante destacar que los resultados presentados corresponden solo a resultados parciales, ya que el presente estudio comprende más años de mediciones. Por lo tanto, recién al finalizar la toma de datos se podrá tener una mejor idea acerca de si el uso silvopastoril afecta o no a la microbiología del suelo.

Palabras clave: Biomasa microbiana, Nothofagus, Carbón.

\footnotetext{
${ }^{4}$ Estación Agropecuaria INTA Santa Cruz gargaglione.veronica@inta.gob.ar

${ }^{5}$ Universidad Nacional de la Patagonia Austral, ICASUR

${ }^{6}$ CONICET
} 


\section{SUMMARY}

Nothofagus antarctica (Nire) is a native species from Patagonian forests that grows from $36^{\circ} 30^{\prime}$ to $56^{\circ} 00^{\prime} \mathrm{SL}$. Nire is in Santa Cruz province (Argentine) the second most abundant forest species.

Nowadays, $90 \%$ of these forests are mainly used as silvopastoral systems, where in the same portion of land coexist trees, natural grasses and cattle or sheep. Organic matter decomposition is an important process made by microorganisms that use detritus as habitat and source of carbon $(\mathrm{C})$ and energy.

The aim of this work was to study biomass of microorganisms in Nothofagus antarctica soils of primary forests and also in forests under silvopastoral use, to detect if this kind of use affect soil biology.

An experimental assay was carried out in two times (spring and summer). Soil samples (5 - $10 \mathrm{~cm}$ depth) were taken in three different farms (Cancha Carreras, Tres Marías and Morro Chico) where $N$. antarctica were growing in both situations (primary forest and silvopastoral use) in South of Santa Cruz province, Argentina.

The fumigation-extraction method was used to detect carbon in microbial biomass. No significant differences were found in microorganism's biomass according to forest use. Likewise, no significant differences were found according to season either. Primary forest had around $1209 \mu \mathrm{g}$ of $\mathrm{C}$ by gram of dry soil, meanwhile silvopastoral forests accounted with $850 \mu \mathrm{g}$ of $\mathrm{C}$.

There are not many antecedents about this kind of studies in South Patagonian forests, so this information became more relevant.

It is possible that the high variability of soil environment could influences to not detect significant differences. Likewise, these are only partial results, since this project include more years of study. Thus, more measurements are going to be made in these sites to ensure if silvopastoral use affects microbial biomass.

Keywords: Microbial biomass, Nothofagus, Carbon. 


\section{INTRODUCCION}

Nothofagus antarctica (ñire) es una especie nativa del bosque andino patagónico que se extiende desde los $36^{\circ} 30^{\prime}$ hasta los $56^{\circ} 00^{\prime}$ de latitud Sur, y en la provincia de Santa Cruz ocupa un total de 159.720 ha, siendo la segunda especie en importancia de la provincia (Peri y Ormaechea, 2013).

El ñire es dentro de los Nothofagus, el que presenta mayor plasticidad ambiental, ocupando desde sitios secos lindantes con la estepa expuesta a fuertes vientos del oeste, hasta sitios con exceso de humedad o inundables como mallines o turberas (Veblen et al., 1996). En los mejores sitios presenta un porte arbóreo bien desarrollado pudiendo alcanzar hasta los $20 \mathrm{~m}$ de altura, mientras que en los sitios menos favorables se desarrolla con un porte más arbustivo de hasta 2 - $3 \mathrm{~m}$ de altura (Donoso et al., 2006).

Esta habilidad del ñire para crecer en distintos ambientes estaría relacionada con su capacidad para redistribuir recursos según la calidad de sitio donde se desarrolla, ya que se observó que en sitios secos aumenta la proporción de biomasa destinada hacia raíces mientras que en sitios de mejor calidad aumenta la proporción de biomasa y recursos destinados hacia la porción aérea (Gargaglione et al., 2013).

Actualmente, el $90 \%$ de estos bosques en Patagonia Sur están siendo utilizados como sistemas silvopastoriles, donde en una misma unidad de superficie coexisten el bosque, el estrato herbáceo y el ganado ovino o vacuno (Peri y Ormaechea, 2013). En este sentido, en el último tiempo se ha generado información acerca de la producción forestal, biomasa y nutrientes en árboles y la productividad del estrato herbáceo (Peri, 2010), y se han realizado estudios para incrementar la productividad del sotobosque con la implementación de especies forrajeras y fertilización (Gargaglione et al., 2013).

Además, se ha investigado las interacciones existentes entre los componentes arbóreo y herbáceo de estos sistemas (Gargaglione et al., 2014). Sin embargo, hasta el momento poco se ha indagado en cuanto a la biología del suelo de estos bosques en su estado natural y bajo las posibles modificaciones que tendrían al ser estos utilizados como sistemas silvopastoriles.

En los ecosistemas terrestres, la descomposición de la materia orgánica es un importante proceso mediado por organismos heterótrofos que utilizan al material orgánico muerto, o detritus, como hábitat y fuente de carbono $(C)$ y energía. La descomposición es un proceso complejo por el cual la materia orgánica es degradada a partículas más pequeñas y a formas solubles de nutrientes que quedan disponibles para la absorción vegetal en parte, y otra parte queda inmovilizada en la biomasa microbiana. Esa desintegración gradual es efectuada por agentes físicos y biológicos (bacterias, hongos, actinomicetos, meso y macrofauna) que realizan la fragmentación (reducción de tamaño), lixiviación (salida de materiales solubles por acción del agua) y mineralización (conversión de una forma orgánica a una inorgánica) de los detritos orgánicos.

Este proceso de descomposición contribuye a la formación de la materia orgánica del suelo (Swift et al., 1979) y es una vía fundamental en el ciclado de nutrientes ya que la mayoría de los nutrientes disponibles del suelo de los bosques derivan de la descomposición de la materia fresca de detritos vegetales y microbios asociados (Visser y Parkinson, 1992).

Numerosos factores pueden influir en el proceso de descomposición, como la temperatura y humedad del ambiente, la composición de la comunidad microbiana y la cantidad y calidad del recurso a descomponer (Couteaux et al., 1995; Aerts, 1997). Asimismo, el componente microbiológico puede servir como indicador del estado general del suelo, pues una alta actividad microbiana es asociada a un buen nivel de fertilidad y constituye un marcador biológico 
potencialmente útil para evaluar las perturbaciones que puedan presentarse. En este sentido, el C contenido en la biomasa microbiana es un componente lábil del pool de la materia orgánica del suelo y es considerado un buen indicador a corto plazo de los efectos de las distintas prácticas del manejo sobre las propiedades biológicas del suelo (Carter and Rennie, 1982; Campbell et al., 1991; Franzluebbers et al., 1994) ya que, por ejemplo, suelos disturbados usualmente contienen menores valores de biomasa microbiana que suelos de bosques y pastizales.

Se considera que el carbono microbiano oscila entre $100-1000 \mu \mathrm{g} / \mathrm{g}$ en suelos arables y entre 500 y 10.000 en suelos forestales (Buscot y Varma, 2005). Estos valores rápidamente decrecen con la profundidad y pueden variar según las distintas estaciones del año (Buscot y Varma, 2005; Gonzalez Polo et al., 2013). La mayor biomasa microbiana suele ser detectada en el detrito caído en bosques y pastizales boreales donde el componente fúngico es el más abundante. Por otra parte, se estima que, independientemente del contenido de $C$ que contenga un suelo en particular, el $\mathrm{C}$ contenido en su biomasa microbiana generalmente comprende alrededor de un 0,9 a $6 \%$ del $\mathrm{C}$ orgánico total, con una media de entre 2 y $3 \%$. Estos valores indican una relación cercana entre el $\mathrm{C}$ microbiano y el $\mathrm{C}$ y nitrógeno $(\mathrm{N}$ ) disponible en los suelos (Buscot y Varma, 2005).

En los bosques de Patagonia existen muy pocos antecedentes de estudios acerca de las características microbiológicas del suelo. Dube et al. (2009) realizaron un trabajo en la zona de

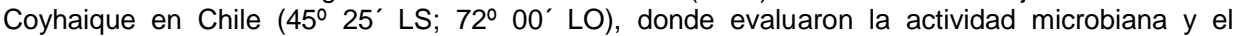
contenido de $\mathrm{C}$ en biomasa microbiana en suelos con distintos tipos de usos: un pastizal natural degradado, un bosque secundario de Nothofagus pumilio y una plantación de Pinus ponderosa.

Estos autores encontraron que la respiración microbiana no varió mucho entre los distintos tratamientos, siendo mayor en la plantación de pino. Asimismo, para el bosque de lenga la respiración varió de $583 \mu \mathrm{g} \mathrm{C}-\mathrm{CO}_{2} / \mathrm{g}$ de suelo seco $\left(0-5 \mathrm{~cm}\right.$ de profundidad) a $59 \mathrm{C}-\mathrm{CO}_{2} / \mathrm{g}$ de suelo seco (10-20 cm de profundidad) mientras que el $C$ en la biomasa microbiana fue de $3705 \mu \mathrm{g} \mathrm{C} / \mathrm{g}$ de suelo seco a los 0-5 cm de profundidad y $139 \mu \mathrm{g} \mathrm{C} / \mathrm{g}$ de suelo seco en el horizonte $10-20 \mathrm{~cm}$ de profundidad.

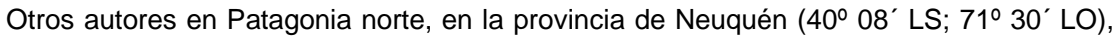
en bosques mixtos de Nothofagus dombeyi, Nothofagus nervosa y Nothofagus obliqua informaron que los de detritos leñosos gruesos (restos de fustes y ramas gruesas de diámetro mayor a los 7,6 $\mathrm{cm}$ ) influían positivamente en los microorganismos del suelo, ya que la actividad enzimática y la descomposición eran mayores bajo los detritos gruesos con estado avanzado de descomposición que en la matriz del suelo, durante la época de verano (Gonzalez Polo et al., 2013).

En los bosques nativos de Patagonia Sur, por su parte, no existen antecedentes de mediciones de actividad microbiana o contenido de $\mathrm{C}$ en biomasa microbiana, aunque sí existen algunos antecedentes de estudios directamente relacionados, como estudios de descomposición y mineralización, tanto en bosques prístinos como bajo uso silvopastoril (Bahamonde et al., 2012; 2013). Por ejemplo, en bosques de Nothofagus antarctica al sur de la provincia de Santa Cruz, la mineralización neta de $\mathrm{N}$ en sitios de clase intermedia era mayor entre las copas de los árboles (nivel de radiación incidente intermedia) que en un sitio abierto sin árboles, mientras que en sitios de peor calidad ocurría el patrón inverso (Bahamonde et al., 2013). Estos autores indicaron que gran parte de las diferencias en los patrones de nitrificación y mineralización en estos bosques bajo distintos regímenes de luz se explicarían en parte por diferencias en el contenido de $\mathrm{C}$ y $\mathrm{N}$ en la biomasa de los microorganismos.

En síntesis, en Patagonia Sur si bien se han realizado estudios con resultados que infieren acerca de las características de la población microbiana, se evidencia la falta de mediciones directas de algunas variables microbiológicas que sirvan como base para el 
entendimiento de los numerosos procesos que se dan en los suelos.

En este contexto, el objetivo del presente trabajo fue evaluar el contenido de biomasa microbiana en bosques de Nothofagus antarctica al sur de Patagonia, bajo diferentes condiciones de uso, bosque primario y bosque silvopastoril, y en dos épocas diferentes, primavera y verano.

Las mediciones están previstas de efectuarse durante dos años consecutivos, y actualmente se encuentran en ejecución, por lo que en este trabajo se presentan los primeros resultados correspondientes al primer año de medición.

\section{MATERIALES Y METODOS}

\section{Sitio de Estudio}

El estudio se realizó en tres estancias diferentes ubicadas en el sudoeste de la provincia

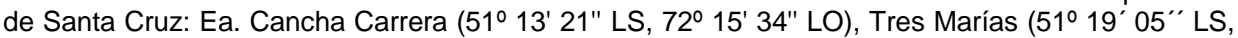
$72^{\circ} 10^{\prime} 47^{\prime \prime}$ LO) y Rincón de los Morros (5157' 24" LS, 71³1' 48" LO). Todas ellas se caracterizan por tener situaciones de bosque primario y bosque bajo uso silvopastoril con ganado.

El clima en toda la zona es templado frío con una temperatura media anual entre 5,5 y $8^{\circ}$ C y una precipitación media anual de alrededor de $550 \mathrm{~mm}$. Los suelos pertenecen al orden Molisoles (haploboroles énticos). Para la caracterización del suelo del sitio y cada situación (bosque sin utilización y bosque bajo uso silvopastoril con ganado) se tomaron 5 muestras al azar compuestas por tres submuestras de $5-10 \mathrm{~cm}$ de profundidad antes del inicio del estudio.

Dichas muestras fueron secadas al aire, tamizadas $(2 \mathrm{~mm})$ y se determinó $\mathrm{pH}$, resistencia de la pasta y textura con el método de Bouyoucos (1962). El pH se determinó en solución acuosa (extracto 1:2 suelo:agua) (Robertson et al., 1999).

Además se determinaron los cationes intercambiables ( $\mathrm{Ca}, \mathrm{K}$ y $\mathrm{Mg}$ ), el contenido de $\mathrm{N}$ total, $\mathrm{C}$ orgánico $(\mathrm{CO})$ y fósforo $(\mathrm{P})$ extraíble.

El contenido de $\mathrm{C}$ y $\mathrm{N}$ total se determinó con el método de combustión seca con un analizador elemental Carbo Erba (Sollins et al., 1999).

El P extraíble en $\mathrm{NaHCO}_{3} 0.5 \mathrm{M}(1: 20$, suelo:solución) se determinó con el método de molibdato ácido ascórbico (Kuo, 1996).

\section{Mediciones de Carbono en Biomasa Microbiana}

En cada sitio de muestreo ( $n=3$ estancias) se tomaron cuatro muestras compuestas por cinco submuestras en los $0-10 \mathrm{~cm}$ de profundidad con un tubo de PVC $(5,6 \mathrm{~cm}$ de diámetro) dos veces al año (inicio de primavera y fin de verano) en las dos situaciones de uso (bosque primario y bajo uso silvopastoril).

Las muestras colectadas fueron mantenidas en heladera $\left(4^{\circ} \mathrm{C}\right)$ hasta su posterior análisis en el laboratorio.

El diseño del experimento corresponde a un diseño factorial con dos factores, condición de uso y estación.

Las estimaciones del $\mathrm{C}$ en biomasa microbiana se realizaron mediante el método de 
fumigación-extracción (Vance et al., 1987). Este método se basa en el aumento de la cantidad de C extractable con $\mathrm{K}_{2} \mathrm{SO}_{4}$ producto de la fumigación (con respecto a la muestra sin fumigar) proveniente del $\mathrm{C}$ lábil liberado a la solución del suelo por la muerte de los microorganismos con los vapores de cloroformo.

Previamente a la cuantificación de $\mathrm{C}$ en biomasa microbiana las muestras de suelo se llevaron a temperatura ambiente y contenido hídrico a capacidad de campo, tomando submuestras de $30 \mathrm{~g}$ para determinación de porcentaje de humedad ya que las determinaciones se deben expresar en base a peso seco.

Luego se tomaron dos submuestras de $50 \mathrm{~g}$ de peso fresco, una como control y otra para fumigar. Las muestras control fueron extraídas con $50 \mathrm{ml}$ de $\mathrm{K}_{2} \mathrm{SO}_{4} 0,5 \mathrm{M}$, y agitadas en un agitador horizontal por 1 hora.

Las muestras a fumigar fueron colocadas en un desecador junto con un recipiente conteniendo $30 \mathrm{ml}$ de cloroformo libre de etanol, el cual se llevó a ebullición mediante bomba de vacío. Luego se dejó reposar por 24 h en oscuridad. Finalizado este período, se hizo la extracción y agitación de la misma forma que a las muestras control.

Las muestras fueron digeridas con solución sulfocrómica por 30 min a $150 \stackrel{\circ}{ } \mathrm{C}$. La concentración de $\mathrm{C}$ de las muestras digeridas se obtuvo mediante la lectura con un espectrofotómetro. Previamente, se realizó una curva de calibración usando biftalato de potasio como patrón.

La conversión de $\mathrm{C}$ a biomasa microbiana se realizó mediante la fórmula:

$\mathrm{C}$ en biomasa microbiana $=(\mathrm{Cf}-\mathrm{Cnf}) / \mathrm{KEC}$

Donde: $\mathrm{Cf} \quad=$ Carbono en el extracto fumigado

Cnf = Carbono en el extracto no fumigado

KEC = Constante de eficiencia de la fumigación $=0,45$ (Jenkinson and Ladd, 1981).

\section{Análisis Estadísticos}

El diseño del experimento corresponde a un diseño factorial con dos factores, uso (bosque primario y silvopastoril) y estación (primavera y verano), con tres repeticiones (tres estancias).

EI C en biomasa microbiana se analizó mediante ANOVAS con el software Infostat 2.0. En caso de encontrar diferencias significativas se realiza el test de Tukey a un nivel de significancia de 0.05 .

\section{RESULTADOS}

El suelo del bosque presentó una textura arenosa con valores de $\mathrm{pH}$ de alrededor de 5,8.

No se encontraron diferencias significativas en las características iniciales del suelo según este sea de bosque silvopastoril o bosque primario, aunque este último presentó valores ligeramente más altos en $\mathrm{K}, \mathrm{C}, \mathrm{y} \mathrm{N}$ y el doble de $\mathrm{P}$ (Cuadro $\mathrm{N}^{\circ} 1$ ). 


\section{Cuadro $\mathrm{N}^{\circ} 1$}

CARACTERÍSTICAS INICIALES DEL SUELO DE LOS SITIOS DE ESTUDIO

\begin{tabular}{|l|c|c|c|c|c|c|}
\hline \multicolumn{1}{|c|}{ Sitio } & $\begin{array}{c}\mathrm{K} \\
(\mathbf{m e q} / 100 \mathrm{~g})\end{array}$ & $\begin{array}{c}\mathbf{C} \\
(\%)\end{array}$ & $\begin{array}{c}\mathbf{N} \\
(\%)\end{array}$ & $\mathbf{C : N}$ & $\begin{array}{c}\mathbf{P} \\
(\mathbf{p p m})\end{array}$ & $\mathbf{p H}$ \\
\hline Bosque Primario & $1.21(0.29)$ & $7.74(2.28)$ & $0.55(0.20)$ & $14.23(1.45)$ & $43.14(3.0)$ & $5.89(0.36)$ \\
Bosque Silvopastoril & $1.09(0.52)$ & $6.57(4.86)$ & $0.50(0.38)$ & $13.03(0.55)$ & $23.24(25.8)$ & $5.84(0.14)$ \\
Significancia(p<0.05) & $\mathrm{ns}$ & $\mathrm{ns}$ & $\mathrm{ns}$ & $\mathrm{ns}$ & $\mathrm{ns}$ & $\mathrm{ns}$ \\
\hline
\end{tabular}

( ): Desvío estándar de la media

ns: No se encontraron diferencias significativas entre los sitios

El suelo del bosque primario presentó mayores valores de biomasa microbiana que el bosque bajo uso silvopastoril, siendo 1.090 y $1.328 \mu \mathrm{g} \mathrm{C} / \mathrm{g}$ de suelo seco para diciembre y febrero respectivamente.

El bosque silvopastoril en tanto presentó 827 y $872 \mu \mathrm{g} \mathrm{C} / \mathrm{g}$ de suelo seco para las mismas fechas (Figura $\mathrm{N}^{\circ} 1$ ).

Sin embargo, estas diferencias no fueron significativas. Tampoco se encontraron diferencias significativas entre épocas del año para un mismo tipo de bosque.
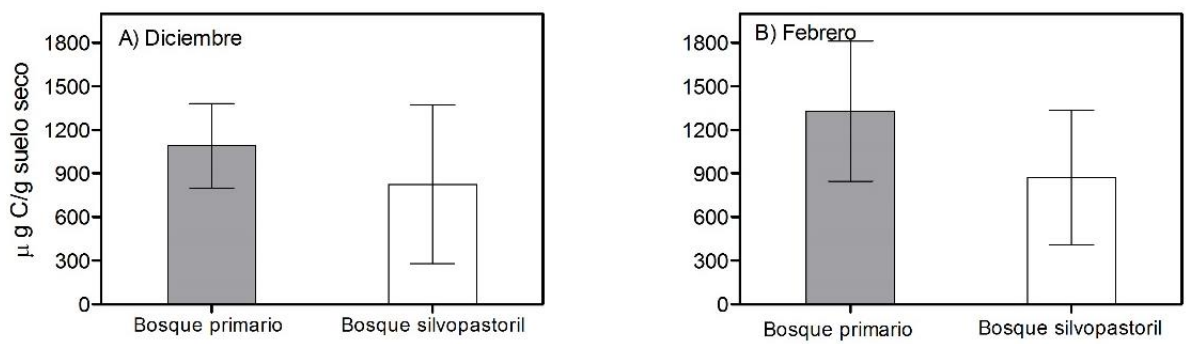

Las barras verticales indican el desvío estándar de la media.

A) Primavera (diciembre) y B) Verano (febrero).

Figura $N^{\circ} 1$

VALORES DE BIOMASA MICROBIANA EN SUELOS DE BOSQUE EN DOS CONDICIONES DE USO DIFERENTES

\section{DISCUSION}

Los valores de biomasa microbiana encontrados en el presente estudio son inferiores a los informados por Gonzalez Polo et al. (2013) para un boque mixto de Nothofagus (N. dombeyi, $N$. nervosa y $N$. obliqua) en Patagonia Norte. Estos autores encontraron que la biomasa microbiana en la matriz del suelo variaba entre 1000 y $2000 \mu \mathrm{g} \mathrm{C} / \mathrm{g}$ de suelo seco para otoño y verano, respectivamente.

Estas diferencias pueden deberse a que al norte de Patagonia la temperatura y las condiciones ambientales en general son más favorables que en el extremo sur. Por otra parte, 
Ross et al. (1996) informaron valores de alrededor de 1.670 microgramo de $\mathrm{C} / \mathrm{g}$ de suelo en los primeros $0-10 \mathrm{~cm}$ de profundidad en bosques de Nothofagus solandri en Nueva Zelanda, los cuales son cercanos a los encontrados en este estudio para el bosque primario (entre $1.000 \mathrm{y}$ $1.300 \mu \mathrm{g} \mathrm{C/g}$ de suelo seco).

Si bien en el presente estudio no se encontraron diferencias significativas entre bosque primario y bosque silvopastoril, es importante destacar que el bosque bajo uso silvopastoril en todos los casos presentó una menor cantidad de biomasa microbiana comparado con el bosque primario. Esta disminución correspondió a un $24 \%$ menos en primavera y a un $34 \%$ menos para el verano.

Otro aspecto a tener en cuenta es que los presentes son resultados preliminares correspondientes al primer año de medición, con lo cual, aún no se puede decir a ciencia cierta si el uso silvopastoril afecta o no a la microbiología de suelo en estos bosques. El presente ensayo comprende al menos dos años consecutivos de toma de datos, por lo que recién al finalizar el estudio se podrá tener una mejor idea en este aspecto.

\section{REFERENCIAS}

Aerts, R., 1997. Climate, leaf litter chemistry and leaf litter decomposition in terrestrial ecosystems: a triangular relationship. Oikos 79: 439-449.

Bahamonde, H. A.; Peri, P. L.; Alvarez, A.; Barneix, A.; Moretto, A.and Martínez Pastur, G., 2012. Litter decomposition in Nothofagus antarctica forests under silvopastoral use in Southern Patagonia. Agroforest Syst 84 , 345-360.

Bahamonde, H. A.; Peri, P. L.; Alvarez, A.; Barneix, A.; Moretto, A. and Martínez Pastur, G., 2013. Silvopastoral use of Nothofagus antarctica in Southern Patagonian forests, influence over net nitrogen soil mineralization. Agroforest Syst 87, 259-271.

Bouyoucos, G. J., 1962. Hydrometer method for making particle size analisis de soils. Agrom. Jor. 54: 464-465.

Buscot, F. and Varma, A., 2005. Microorganisms in soils: Roles in Genesis and Functions. Springer- Berling Springer-Verlag Berlin Heidelberg. ISBN: 3-540-22220-0. Germany.

Campbell, C. A.; Biederbeck, V. O.; Zentner, R. P. and Lafond, G. P., 1991. Effect of crop rotations and cultural practices on soil organic matter, microbial biomass and respiration in a thin Black Chernozem. Can. J. Soil Sci. 71, 363-376.

Carter, M. R. and Rennie, D. A., 1982. Changes in soil quality under no-tillage farming systems: distribution of microbial biomass and mineralizable $\mathrm{C}$ and N potential. Can. J. Soil Sci. 62, 587-597.

Couteaux, M. M.; Botter, P. and Berg, B., 1995. Litter decomposition, climate and litter quality. Trends Ecol Evol 10:63-66.

Donoso, C.; Steinke, L. y Premoli, A., 2006. Nothofagus antarctica. Pp. 401-410 en: Donoso, C. (Ed.). Las especies arbóreas de los bosques templados de Chile y Argentina. Autoecología. Marisa Cuneo Ediciones, Valdivia, Chile.

Dube, F.; Zagal, E.; Stolpe, N. and Espinosa, M., 2009. The influence of land-use change on the organic carbon distribution and microbiol respiration in a volcanic soil of the Chilean Patagonia. Forest Ecol Manag 257, 169951704.

Franzluebbers, A. J.; Hons, F. M. and Zuberer, D. A., 1994. Long-term changes in soil carbon and nitrogen pools in wheat Management systems. Soil Sci. Soc. Am. J. 58, 1639-1645.

Gargaglione, V.; Peri, P. L. y Rubio, G., 2013. Partición diferencial de nutrientes en árboles de Nothofagus 
antarctica creciendo en un gradiente de calidades de sitio en Patagonia Sur. Bosques 34 (3): $291-302$.

Gargaglione, V.; Peri, P. L. and Rubio, G., 2014. Tree-grass interactions for N in Nothofagus antarctica silvopastoral systems: Evidence of facilitation from trees to underneath grasses. Agroforestry systems (in press) DOI: DOI 10.1007/s10457-014-9724-3.

González-Polo, M.; Fernández-Souto, A. and Austin, A. T., 2013. Coarse woody debris stimulates soil enzimatic activity and litter decomposition in an old growth temperate forest of Patagonia, Argentina. Ecosystems 16: 1025-1038.

Jenkinson, D. S. and Ladd, J. N., 1981. Microbial biomass in soil: Measurement and turnover. In Soil Biochemistry, Vol. 5 (E. A. Paul and J. N. Ladd, Eds.), pp. 415-471. Dekker, New York.

Kuo, S., 1996. Phosphorus. Pages 869-919 in D. L. Sparks, A. L. Page, P. A. Helmke, R. H., Loeppert, P. N. Soltanpour, M. A. Tabatabai, C. T. Johnston, and M. E. Sumner, editors. Methods of Soil Analysis. Part 3. Chemical methods. Soil Science Society of America, Madison, WI.

Peri, P. L., 2010. Sistemas silvopastoriles en bosques de Nothofagus antarctica: Revisión del conocimiento actual en Patagonia Sur, Argentina. Actas I Congreso Internacional Agroforestal Patagónico, 15 pp. Coyhaique, Chile, 27 al 29 de Octubre de 2010.

Peri, P. L. y Ormaechea, S., 2013. Relevamiento de los bosques nativos de ñire (Nothofagus antarctica) en Santa Cruz: Base para su conservación y manejo, 88 pp. Ediciones INTA, Buenos Aires. ISBN 978-987-679-219-6

Robertson, G. P.; Wedin, D.; Groffman, P. M.; Blair, J. M.; Holland, E.; Nadelhoffer, K. J. and Harris D.,1999. Soil Carbon and nitrogen availability nitrogen mineralization, nitrification and soil respiration potentials. In: Standard soil methods for long term ecological research. Robertson, G. P.,

Coleman, D.C. Bledsoes, C. S and Sollins, P. (Eds.). Oxford University Press, New York.

Ross, D. J.; Tate, K. R. and Feltham, C. W., 1996. Microbial biomass, and C and N mineralization, in litter and mineral soil of adjacent montane ecosystems in a southern beech (Nothofagus) forest and a tussock grassland. Soil Biol. Biochem. Vol.28, No. 12, pp. 1613-1620.

Sollins, P.; Glassman, C.; Paul, E. A.; Swanston, C.; Lajtha, K.; Heil, J. W. and Elliott, E. T., 1999. Soil carbon and nitrogen pools and fractions. Páginas 89-105 en P. G. Robertson, D. C. Coleman, C. S. Bledsoe, y P. Sollins, editores. Standard soil methods for long-term ecological research. Oxford University Press, New York.

Swift, M. J.; Heal, O. W. and Anderson, J. M., 1979. Decomposition in terrestrial ecosystems. Studies in Ecology 5. Univ. California Press. Berkeley y Los Ángeles.

Vance, E. D.; Brookes, P. C. and Jenkinson, D. S., 1987. An extraction method for measuring soil microbial biomass. Soil Biol Biochem 19:703-707.

Veblen, T. T.; Donoso, C.; Kitzberger, T. and Rebertus, A. J., 1996. Ecology of southern Chilean and Argentinean Nothofagus forests. Pp. 293-353 en: Veblen, T., Hill, R., Read, J. (Eds.) The Ecology and Biogeography of Nothofagus Forests, Yale University Press, New Haven.

Visser, S. and Parkinson, D., 1992. Soil biological criteria as indicators of soil quality: soil microorganisms. Am. J. Alt. Agric. 7(1-2), 33-37. 
